\title{
A Rare Simultaneous Occurrence of Appendiceal Diverticulitis and Peptic Ulcer that Leads to Abdominal Peritonitis Symptoms
}

\author{
Maziar Moayerifar ${ }^{1}$, Hossein Torabi ${ }^{1}$, Kasra Shirini ${ }^{2}$, and Yalda Ashoorian ${ }^{1}$ \\ ${ }^{1}$ Guilan University of Medical Sciences \\ ${ }^{2}$ Iran University of Medical Sciences
}

March 5, 2022

\begin{abstract}
Appendiceal diverticulitis is a rare condition that can mimic other diseases' symptoms or cause different symptoms because of its various complications. However, one of the most frequent complications of this disease is perforation, leading to other serious problems such as peritonitis. This complication can threaten a patient's health condition.
\end{abstract}

A Rare Simultaneous Occurrence of Appendiceal Diverticulitis and Peptic Ulcer that Leads to Abdominal Peritonitis Symptoms

Authors: Maziar Moayerifar ${ }^{1}$.MD, Hossein Torabi. MD $^{2}$, Kasra Shirini. MD ${ }^{3 *}$, Yalda Ashoorian.MD ${ }^{4}$

Department of General Surgery, Poursina Medical and Educational Center, Guilan University of Medical Sciences, Rasht, Guilan, Iran

Email: dr_maziar_m@yahoo.com

Department of General Surgery, Poursina Medical and Educational Center, Guilan University of Medical Sciences, Rasht, Guilan, Iran

Email: dr.torabi1367@yahoo.com

Department of General Surgery, Iran University of Medical Science, Tehran, Iran, Email: kasrashirini21@gmail.com

Department of Pathology and Laboratory Medicine, Rasht, Guilan University of Medical Science, Rasht, Iran, Email: Y.ashoorian@gmail.com

Corresponding Author: Kasra Shirini, MD, Iran University of Medical Science, Tehran, Iran Email: Kasrashirini21@gmail.com Telephone number: 00989112481500 P.O Box: 14665-354

Acknowledgment: None

Figures: 6 Tables: 0

Abstract Word Count: 94

Main article Word Count: 1440

Disclosure of conflicts of interest: The authors certify that there is no conflict of interest with any financial organization regarding the material discussed in the manuscript. The patient has consented to the submission of the case report for submission to the journal. 
Funding: The authors report no involvement in the research by the sponsor that could have influenced the outcome of this work.

Authors' contributions: All authors contributed equally to the manuscript and read and approved the final version of the manuscript.

Patient's contest: Written informed consent was obtained from the patient to publish this report in accordance with the journal's patient consent policy.

Abstract:

Appendiceal diverticulitis is an infrequent disease that can mimic other diseases' symptoms or cause different symptoms because of its various complications. However, one of the most frequent complications of this disease is a perforation that can lead to other serious problems such as peritonitis. This complication can threaten a patient's health condition. In this article, a male patient presented with abdominal pain and was admitted to the surgical ward with suspicion of a perforated peptic ulcer. However, more investigation showed a simultaneous occurrence of peptic ulcer and perforated appendiceal diverticulitis that cause peritonitis symptoms.

Keywords: appendiceal diverticula, appendiceal diverticulitis, peritonitis, peptic ulcer, laparotomy

Introduction:

The first person who described Appendiceal diverticulitis was Kelynack, a pathologist in the 19th century. He described it as "a greatly distended appendix, totally shut off from the cecum, having two distinct diverticular processes directed between the folds of the mesentery" and classified it into congenital and acquired types [1,2]. It is a rare condition with a prevalence incidence of $0.014 \%$ to $1.9 \%$ [2-4]. Studies showed that despite the similarities between appendiceal diverticulitis and acute appendicitis, the prevalence of perforation in appendiceal diverticulitis is three to four times higher which increases the risk of abdominal peritonitis. Also, it can be synchronous neoplasms such as carcinoids and mucinous adenomas and adenocarcinomas, or it can mimic other diseases' symptoms. So the preoperative diagnosis of this disease is challenging and essential $[2,5]$. As regards this issue, a patient with generalized abdominal peritonitis symptoms, without any specific appendicitis symptoms, presented in this article and finally discovered appendiceal diverticulitis as the main reason but mimicking abdominal peritonitis due to perforated peptic ulcer.

Case presentation:

A 35-year-old patient presented to the surgical department of Poursina Hospital Medical Center, Rasht, Iran, in October 2021, with no history of underlying diseases and with complained of abdominal pain started five days ago with a predominance of the left lower quadrant of the abdomen and hypogastric area and two times vomiting, a day before hospitalization. The patient claimed that the abdominal pain had been vague and generalized and started five months ago for the first time. It was persistent but improved with the use of painkillers. The pain was worsened with feeding and relatively relieved by lying supine, and it had nothing to do with defecation and gas passing. The patient was a drug abuser. He also mentioned occasional constipation from the beginning of the pain in the past five years ago. Moderate but progressive generalized tenderness was detected during physical examination but guarding, and rebound tenderness were not detected. He had a low-grade fever, estimated at 38.1 Celsius, but other vital signs were normal. Therefore, due to the patient's symptoms, he was admitted to the surgical ward with suspicion of general peritonitis due to the perforated peptic ulcer. He was asked to do an upright chest X-ray and supine abdominal X-ray. The upright chest X-ray pictures were unrevealing, as shown in Figure 1. As shown in Figure 2, dilated, gas-filled bowel loops in the supine abdominal X-ray could signify mechanical or ileus obstruction. Furthermore, the Rigler sign can be seen in the abdominal X-ray that was a sign of pneumoperitoneum, leading the surgical team to reinforce the suspicion of perforation. He was also asked to do an endoscopic procedure, and the results showed erosive gastropathy and duodenal ulcer, and the patient was prescribed high-dose proton pump inhibitors (PPI). After that, he was asked to do abdominal sonography. The result revealed a blind loop in the right lower quadrant (RLQ) of the abdominal cavity with standard 
size in the base and proximal parts. Still, it increased the diameter in the Tip that it was approximately seven millimeters. It was non-compressive and with slight fat haziness around it. These findings led to suspicion of appendicitis. Due to the inconsistency of the clinical findings during the physical examination with the ultrasonography findings and due to the suspicion of perforated or complicated appendicitis, he was advised to do abdominal computed tomography $(\mathrm{CT})$ scan with intravenous and oral contrast for more investigations. The CT-scan imaging confirmed a mass-like lesion in the RLQ of the abdominal cavity that could represent appendicitis and tissue wall, thickening in the AP region. Still, unlike usual appendicitis, the IV contrast fluid filled the appendix, as shown in Figure 3. The blood test analyzed presented a standard range of lactate dehydrogenase $(\mathrm{LDH})=309$ (usually should be under 460 in adults) and leukocytosis (white blood cells $[\mathrm{WBC}]=11200 \mathrm{~g} / \mathrm{dL}$ with a neutrophilia ratio of $73 \%$ ) and Amylase $=54 \mathrm{U} /$ Lit (normally should be under 95). Unfortunately, generalized abdominal tenderness intensified during hospitalization, and hypogastric rebound tenderness was found as a new sign.

Therefore, the patient underwent laparotomy with suspicion of general peritonitis due to the perforated peptic ulcer or perforated appendicitis. A midline incision was performed. On the external side of the duodenum wall, the stiff tissue was touched at the same site as an ulcer reported by endoscopy but no perforation was detected. Also, a mass-like lesion was seen in the right lower quadrant area of the abdomen cavity with lots of adhesions to its around tissues. It was discreetly removed from the surrounding tissues, and a diverticular appendix appeared, as can be seen in Figure 4. The tissue was sent for more pathological investigations. After providing the necessary hemostasis, the abdominal cavity was closed. He had a good recovery, and the vital signs were stable after surgery. The patient was transferred to the ICU ward and transferred to the surgical ward after one day and discharged after four days with good general condition and stable vital signs. Ciprofloxacin and Metronidazole treatment was started for him and continued for seven days. There was no complication in the three-month follow-up. The pathological reporting showed herniation of mucosa and submucosa and muscular layer through the wall of appendix and confirmed the diagnosis of the appendix with multiple diverticulosis and appendiceal diverticulitis, as can be seen in Figures 5 and 6 .

\section{Discussion:}

Different cases of appendiceal diverticula with different symptoms and complications have been reported, including patients of various ages and with different health conditions $[2,6,7]$. This disease is classified into two groups based on the number of layers herniating through the appendix wall. Acquired or pseudodiverticula form and congenital or true form, which means all three appendiceal layers herniate through a normal wall histologically $[2,4,8]$. The congenital type is rare and accounts for $3 \%$ of all diagnosed appendix diverticulosis cases [9-11]. Appendiceal diverticulitis risk factors are male gender, age over 30, cystic fibrosis, and Hirschsprung disease [12]. Appendiceal diverticulitis is not only a rare condition that can mimic other diseases' symptoms, and most of all, it is confused with acute appendicitis, but also it can be synchronous with other serious diseases such as carcinoid tumors $[2,5]$. One of the most complications of appendiceal diverticulitis is perforation, with an incidence prevalence of $66 \%$ [13]. Other complications could be chronic pain and acute inflammation [14]. Due to these reasons, it is crucial to diagnose this problem preoperatively. Using imaging methods such as ultrasonography and CT-scan can be very useful to diagnose appendiceal diverticulitis, while CT-scan is better and has $80 \%$ sensitivity and $100 \%$ specificity. Still, both methods are highly dependent on radiologists' experiences $[10,13]$. On the other hand, the diagnosis of appendiceal diverticulitis may not be possible due to the small size or involvement of inflammatory mass [12]. So, the definitive diagnosis way is a postoperative pathology report [15].

The definitive treatment way to eradicate symptomatic appendiceal diverticulitis is an appendectomy, and choosing the appropriate surgical method between laparotomy or laparoscopy depends on the patient's condition and surgical team decision [15] Choosing the appropriate surgical method is crucial to perform a safe way to avoid rupture that it can lead to peritoneal seeding and peritonitis consequently [5].

In this article, the male patient presented with abdominal pain and generalized abdominal tenderness with suspicion of perforated peptic ulcer. After medical investigation, such as physical examination, blood test 
analysis, ultrasonography, and endoscopy procedure, the patient was diagnosed with a non-perforated duodenum ulcer and appendicitis. These diagnoses justified the patient's symptoms as the simultaneous occurrence of peptic ulcer and appendicitis can cause generalized abdominal pain and generalized abdominal tenderness. However, as a rebound tenderness suddenly appeared in the physical examination and the patent's abdominal tenderness progressed from moderate to severe, the surgical team decided to choose the laparotomy method instead of the laparoscopy one due to suspicion of general peritonitis and the patient's condition.

\section{Conclusion:}

This article is about a 35-year-old male presenting with generalized abdominal pain with a predominance of the left lower quadrant of the abdomen and hypogastric area mimicking perforated peptic ulcer but found to have abdominal peritonitis due to appendiceal diverticulitis. It is crucial to diagnose and treat this disease preoperatively as some studies showed that appendiceal diverticulitis could be asymptomatic until getting infected or accidentally during a medical investigation or can mimic other diseases' symptoms or occur simultaneously with other serious diseases. So, using different imaging methods such as ultrasonography and CT-scan could be beneficial, but physical examination findings should be considered too. However, the definitive way to diagnose the disease is postoperative pathological investigation. Therefore, appendiceal diverticulitis should be regarded as an important differential diagnosis in patients with abdominal pain.

Disclosure of conflicts of interest: The authors certify that there is no conflict of interest with any financial organization regarding the material discussed in the manuscript. The patient has consented to the submission of the case report for submission to the journal.

Funding: The authors report no involvement in the research by the sponsor that could have influenced the outcome of this work.

Authors' contributions: All authors contributed equally to the manuscript and read and approved the final version of the manuscript.

References:

1. Albeeshi MZ, Alwanyan AA, Salim AA, Albabtain IT: Appendiceal diverticulitis presenting as acute appendicitis diagnosed postoperatively. J Surg Case Rep. 2019, 11:1-3. 10.1093/jscr/rjz332

2. Abdulmomen AA, Zalzahrani AS, Al Mulla LA, Alaqeel FO: Acute perforated appendicitis associated with appendiceal diverticulitis in a young man: a case report with literature review. Am J Case Rep. 2022, 23:e934838-1 - 7. 10.12659/AJCR.934838

3. Williams JM, Adamo DA, Olson MC: Acute appendiceal diverticulitis: a case report. Radiol Case Rep. 2021, 24:1072-1074. 10.1016/j.radcr.2021.02.034

4. Altieri ML, Piozzi GN, Salvatori P, Mirra M, Piccolo G, Olivari N: Appendiceal diverticulitis, a rare relevant pathology: presentation of a case report and review of the literature. Int J Surg Case Rep. 2017, 33:31-34. 10.1016/j.ijscr.2017.02.027

5. Khan SL, Siddeswarappa M, Khan MF: A case report describing diverticulosis of the appendix presenting as acute appendicitis. Int J Surg Case Rep. 2016, 29:155-157. 10.1016/j.ijscr.2016.10.074

6. Souferi B, Sheppard K, Onayemi AO, Davis JM: Incidental findings of appendiceal diverticulitis presenting as acute appendicitis. The American Surgeon. 2021, 10.1177/00031348211065125

7. Krzak AM, Townson A, Malam Y, Mathews J: Diverticulitis complicated by colovenous fistula formation and pylephlebitis. Journal of Surgical Case Reports. 2022, 2022:1-3. 10.1093/jscr/rjab591

8. Dupre MP, Jadavji I, Matshes E, Urbanski SJ: Diverticular disease of the vermiform appendix: a diagnostic clue to underlying appendiceal neoplasm. Hum Pathol. 2008, 39:182318266. 10.1016/j.humpath.2008.06.001

9. Abdullgaffar B: Diverticulosis and diverticulitis of the appendix. Int J Surg Pathol. 2009, 17:231237. $10.1177 / 1066896909332728$

10. Majeski J: Diverticulum of the vermiform appendix is associated with chronic abdominal pain. Am J of Surg. 2003, 186:129-131. 10.1016/S0002-9610(03)00187-9 
11. Konen O, Edelstein E, Osadchi A, Shapiro M, Rathaus V: Sonographic appearance of an appendiceal diverticulum. J Clin Ultrasound. 2002, 30:45-47. 10.1002/jcu.10023

12. Chia ML, Chan SWY, Shelat VG: Diverticular Disease of the Appendix Is Associated with Complicated Appendicitis. GE Port J Gastroenterol. 2021, 28:236-242. 10.1159/000511822

13. Zubieta-O'Farrill G, Guerra-Mora JR, Gudiño-Chávez A, Gonzalez-Alvarado C, Cornejo-López GB, Villanueva-Sáenz E: Appendiceal diverticulum associated with chronic appendicitis. Int J Surg Case Rep. 2014, 5:961-963. 10.1016/j.ijscr.2014.10.066

14. Medlicott SAC, Urbanski SJ: Acquired Diverticulosis of the Vermiform Appendix: A Disease of Multiple Etiologies: A Retrospective Analysis and Review of the Literature. International Journal of Surgical Pathology. 1998, 6:23-27. 10.1177/106689699800600106

15. Fiordaliso M, De Marco AF, Constantini R: A case of type 2 appendiceal diverticulum perforated and a review of the literature. Int J of Sur Case Rep. 2020, 77:450-453. 10.1016/j.ijscr.2020.10.114

Figures Description:

Figure1: Upright Chest X-ray

Figure2: Supine abdominal X-ray, red arrows show gas filled bowel loops, the green arrow points to Rigler sign

Figure3: Abdominal CT-scan, the green arrow shows appendix filled by IV contrast fluid

Figure4: Appendix after appendectomy

Figure5: Microscopic view of diverticular appendix tissue

Figure6: Microscopic view of diverticular appendix tissue 


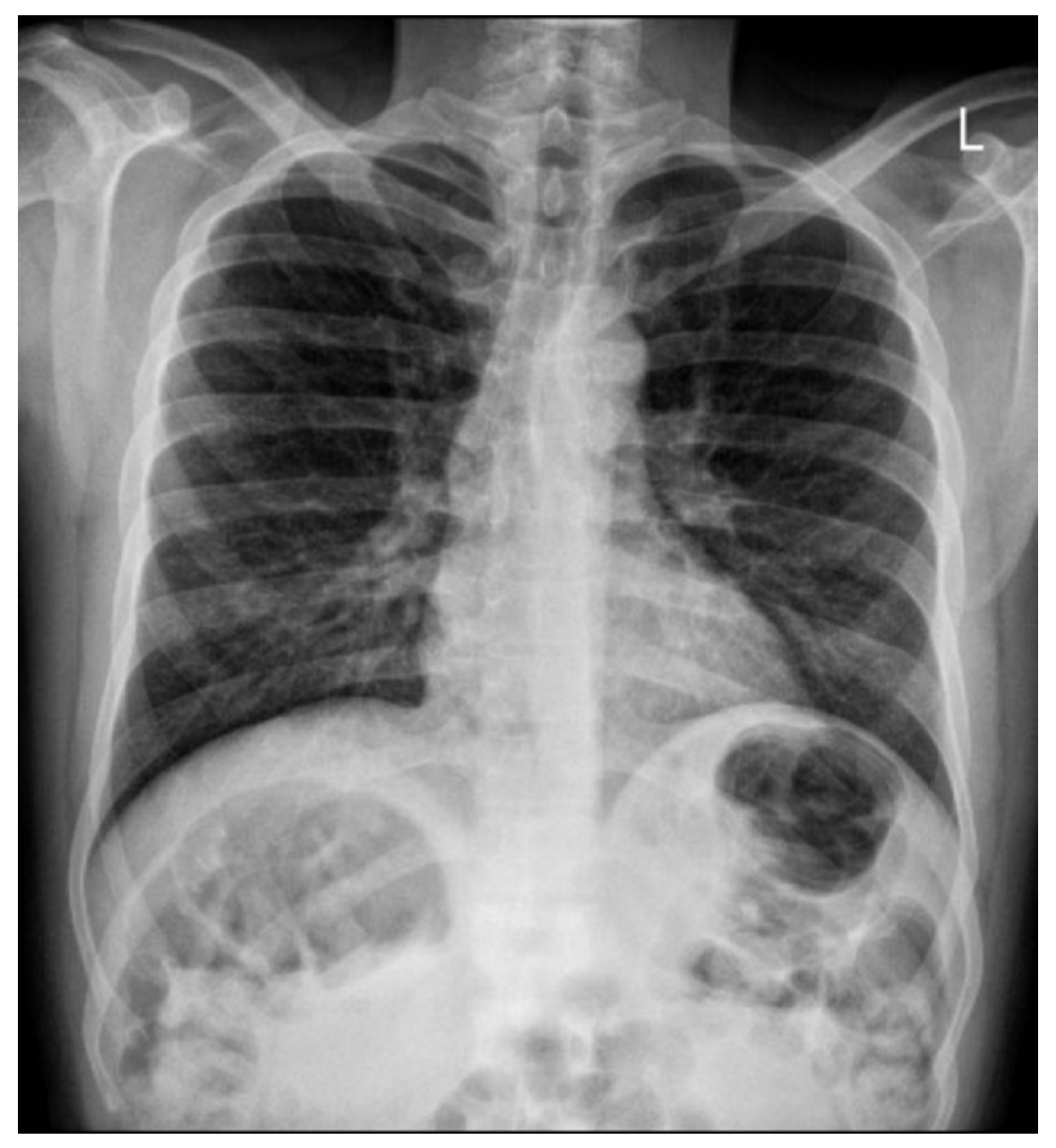



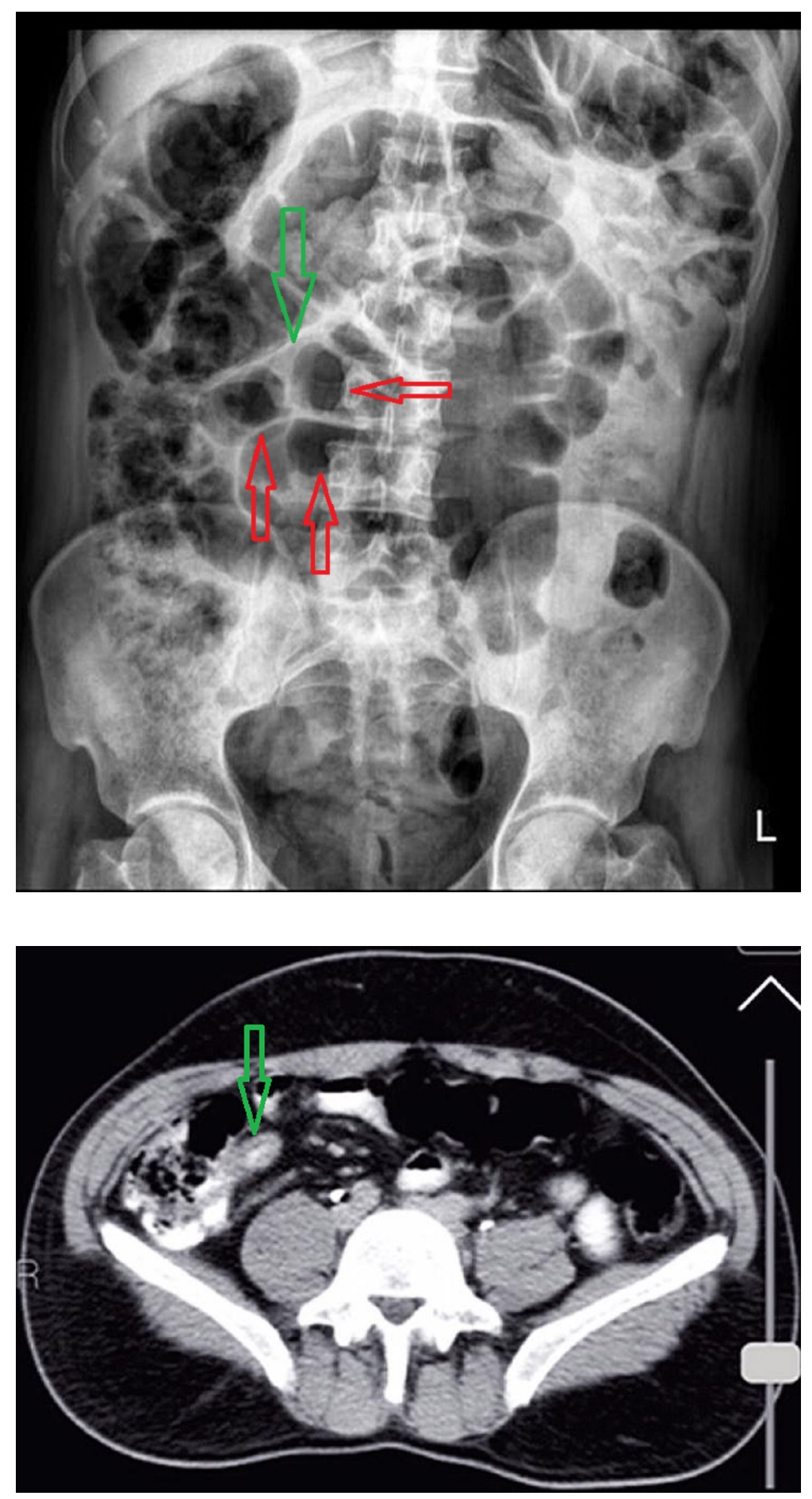


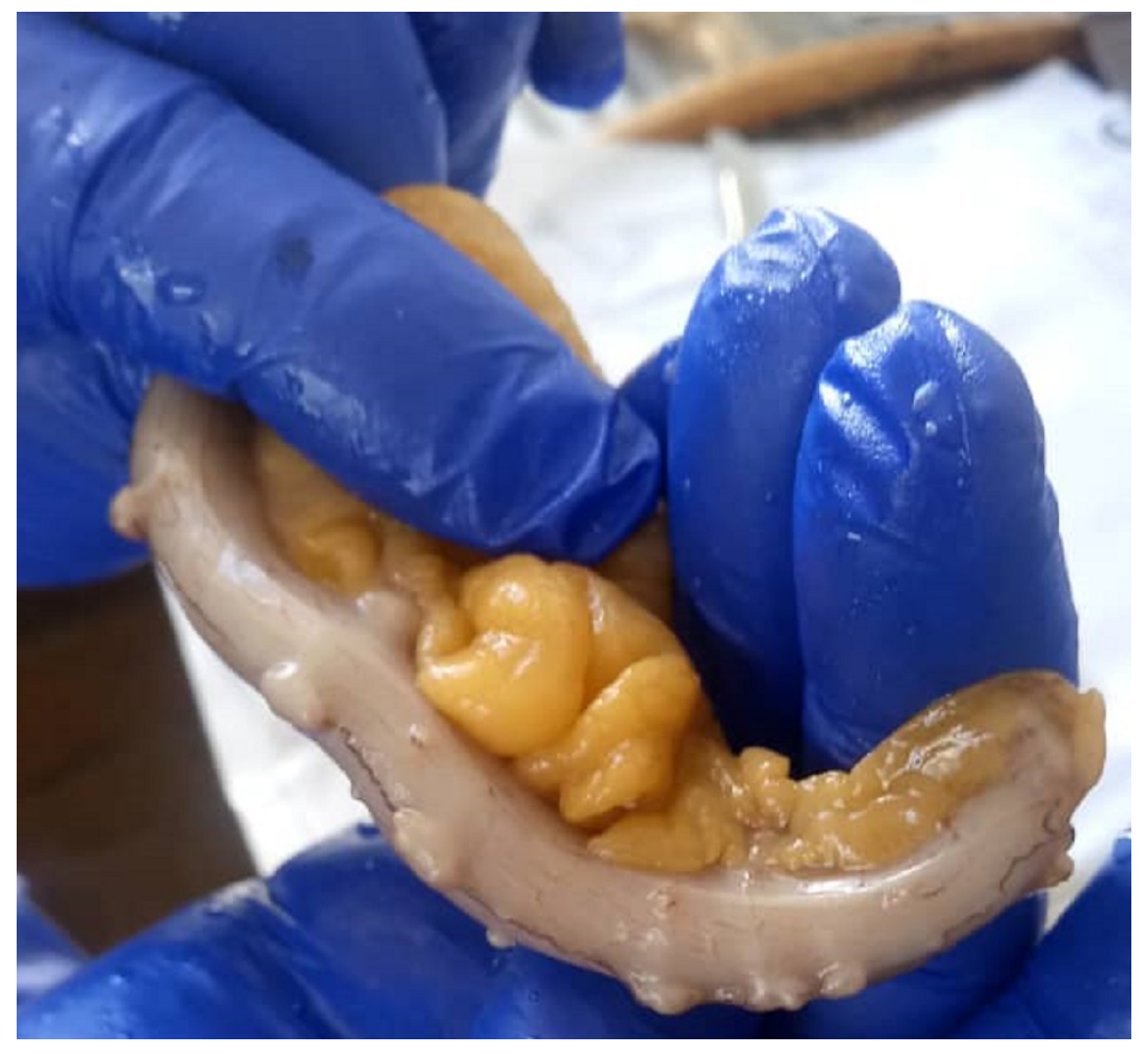




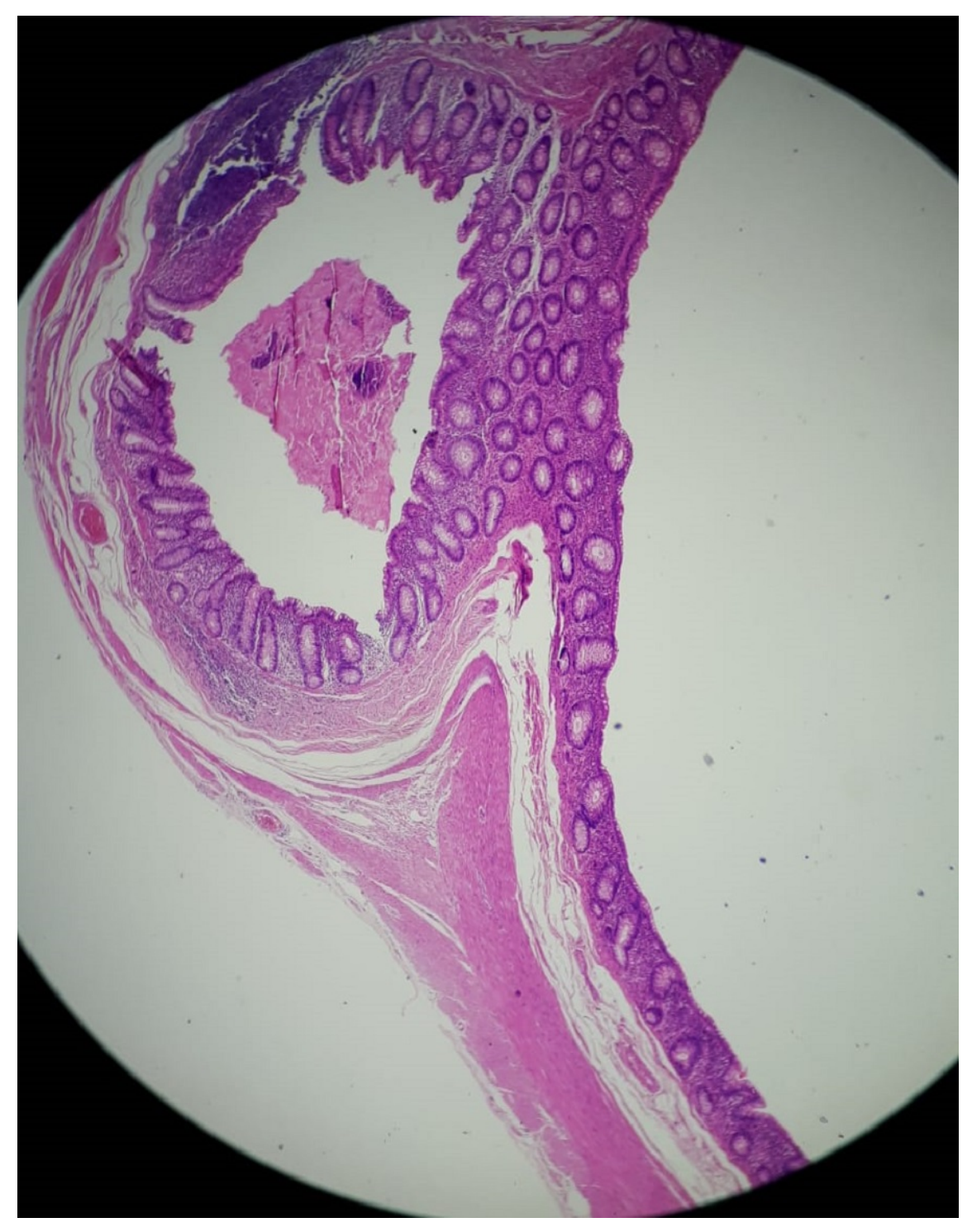




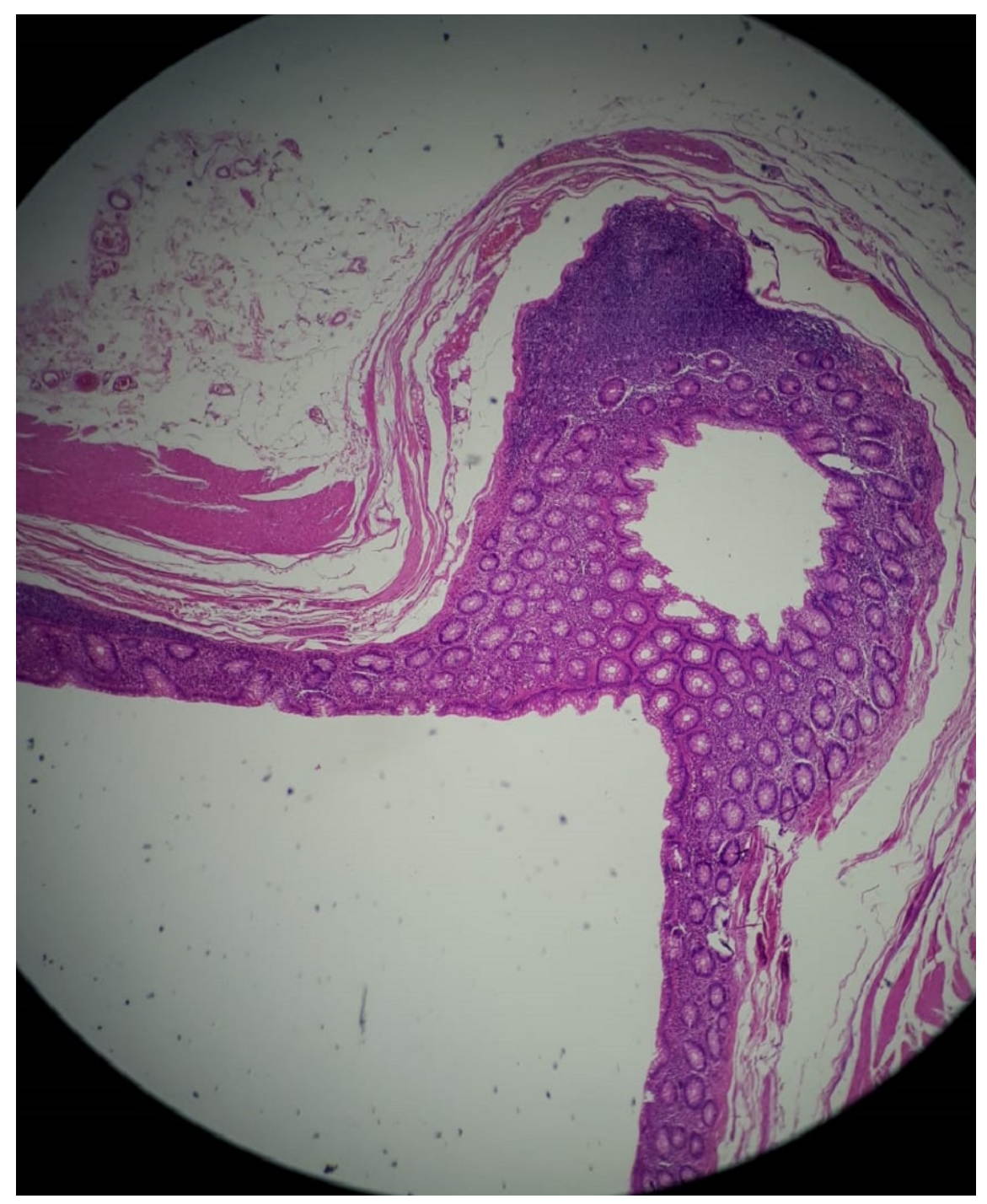

